\title{
Colgajos en hélice de las arterias perforantes para la reconstrucción de los defectos de cubrimiento en la extremidad inferior: experiencia de la IPS Universitaria, Medellín, Colombia
}

\author{
Carolina Posso ${ }^{1}$, Germán WolfF² ${ }^{2}$ Esteban Cardona ${ }^{3}$
}

Palabras clave: colgajo perforante; extremidad inferior; heridas y lesiones; comorbilidad; complicaciones posoperatorias; necrosis.

\section{Resumen}

Introducción. Los colgajos en hélice de las arterias perforantes son una opción reconstructiva regional y versátil para los defectos del miembro inferior.

Materiales y métodos. Se trata de un estudio retrospectivo de cohorte, de 37 colgajos en 31 pacientes, hechos de agosto de 2012 a julio del 2015. Se recolectó la información demográfica, y de los factores perioperatorios, las complicaciones y los resultados.

Resultados. La mediana de la edad de los pacientes fue de 44,3 años (rango: 18 a 82). La causa más común de

1 Médica, cirujana plástica, especialista en Microcirugía, Servicio de Cirugía Plástica y Reconstructiva, Universidad de Antioquia, Medellín, Colombia

2 Médico, cirujano plástico, especialista en Microcirugía, Clínica Las Américas, Medellín, Colombia

3 Médico, residente de Cirugía Plástica, Servicio de Cirugía Plástica y Reconstructiva, Universidad de Antioquia, Medellín, Colombia

Fecha de recibido: 25 de abril de 2017

Fecha de aprobación: 8 de septiembre de 2017

Citar como: Posso C, Wolff G, Cardona E. Colgajos en hélice de las arterias perforantes para la reconstrucción de los defectos de cubrimiento en la extremidad inferior: experiencia de la IPS Universitaria, Medellín, Colombia. Rev Colomb Cir. 2017;32:290-96. los defectos fue el trauma (32\%), aunque las úlceras por presión y la osteomielitis también se presentaron con frecuencia. En cuanto a las comorbilidades, el $29 \%$ de los pacientes presentaba hipertensión arterial sistémica, el $19 \%$, diabetes mellitus, y el $6 \%$, enfermedad arterial oclusiva crónica. Se presentaron dos casos de necrosis total (5\%) y 9 casos de necrosis parcial (24\%). La tasa de necrosis fue mayor en los pacientes con hipertensión arterial sistémica, con enfermedad arterial oclusiva crónica o con un puntaje de 3 en el sistema de clasificación de la American Society of Anesthesiologists (ASA), con una relación estadísticamente significativa.

En conclusión, los colgajos en hélice de las arterias perforantes son un método de cubrimiento de gran versatilidad y confiable para tratar defectos de tejidos blandos del miembro inferior, con tasas de complicaciones similares a las de otros métodos tradicionales de cobertura.

\section{Introducción}

La reconstrucción de los tejidos blandos de la pierna continúa siendo un reto para el cirujano plástico reconstructivo. Existen múltiples opciones disponibles y la tasa de complicaciones continúa siendo alta, especialmente en presencia de comorbilidades ${ }^{1-3}$. Los colgajos regionales, en general, son una excelente opción porque la morbilidad, el tiempo quirúrgico y los costos son menores ${ }^{4}$. 
En la evolución de los colgajos regionales, los colgajos en hélice de las arterias perforantes abrieron nuevas posibilidades de cubrimiento sin necesidad de sacrificar ejes vasculares mayores, ni estar restringidos a un territorio vascular preestablecido ${ }^{5}$.

El propósito de esta revisión es presentar nuestra experiencia con el uso de los colgajos en hélice de las arterias perforantes para la reconstrucción de defectos de cobertura en el miembro inferior.

\section{Pacientes y métodos}

Se llevó a cabo un estudio retrospectivo de cohorte entre agosto de 2012 y julio de 2015, en el cual se incluyeron los pacientes operados en la IPS Universitaria, sede León XIII, que presentaban defectos de tejidos blandos en el miembro inferior, los cuales fueron tratados con colgajos en hélice de las arterias perforantes. Se excluyeron los pacientes que presentaban defectos de cubrimiento en la zona de apoyo plantar.

La variable de exposición se definió como el cubrimiento del defecto con el colgajo y, la variable de resultado, como la presencia de necrosis; esta última, a su vez, se categorizó como necrosis parcial o total. Además, se recolectaron las siguientes variables: edad, sexo, presencia de comorbilidades (tabaquismo, diabetes mellitus, ateroesclerosis e hipertensión arterial sistémica, entre otras), puntaje en la clasificación de la American Society of Anesthesiologists (ASA), etiología y tamaño del defecto, tipo y tamaño del colgajo, arco de rotación, tipo de cierre del sitio donante, complicaciones (necrosis parcial y total) y seguimiento posoperatorio.

\section{Técnica quirúrgica}

Durante el diseño preoperatorio, se utiliza el transductor manual para ecografía Doppler para localizar la arteria perforante más cercana al defecto, en relación con los ejes vasculares mayores de la extremidad ${ }^{6}$. Posteriormente, se continúa con la marcación del resto de la isla. Usualmente, se comienza con una incisión anterior en uno de los bordes para identificar primero la arteria perforante más cercana al defecto. Una vez localizada, se valora su calidad (diámetro, longitud y pulso) y, si se considera adecuada, se termina la incisión de los otros bordes de la isla de piel. Posteriormente, la disección de la arteria perforante se hace utilizando amplificación con lupas e instrumentos de microciru- gía y, así, permitir una rotación libre y sin tensión de la misma, al rotar el colgajo hacia la zona del defecto que se va a cubrir.

Frecuentemente, se encuentra más de una arteria perforante; en estos casos, se utilizan pinzas vasculares, dejando sin ocluir solo la arteria perforante escogida, y se evalúa el sangrado de la dermis. Si la perfusión es adecuada, se pueden ligar las otras arterias perforantes y se continúa con la disección del colgajo. En algunos casos, cuando se encuentran dos arterias perforantes continuas en el mismo eje, se conservan ambas si al rotar el colgajo no se observa limitación. Si se identifica algún problema con la perfusión del colgajo, se debe evaluar otra arteria perforante o, incluso, se debe considerar otra opción reconstructiva (figura 1).
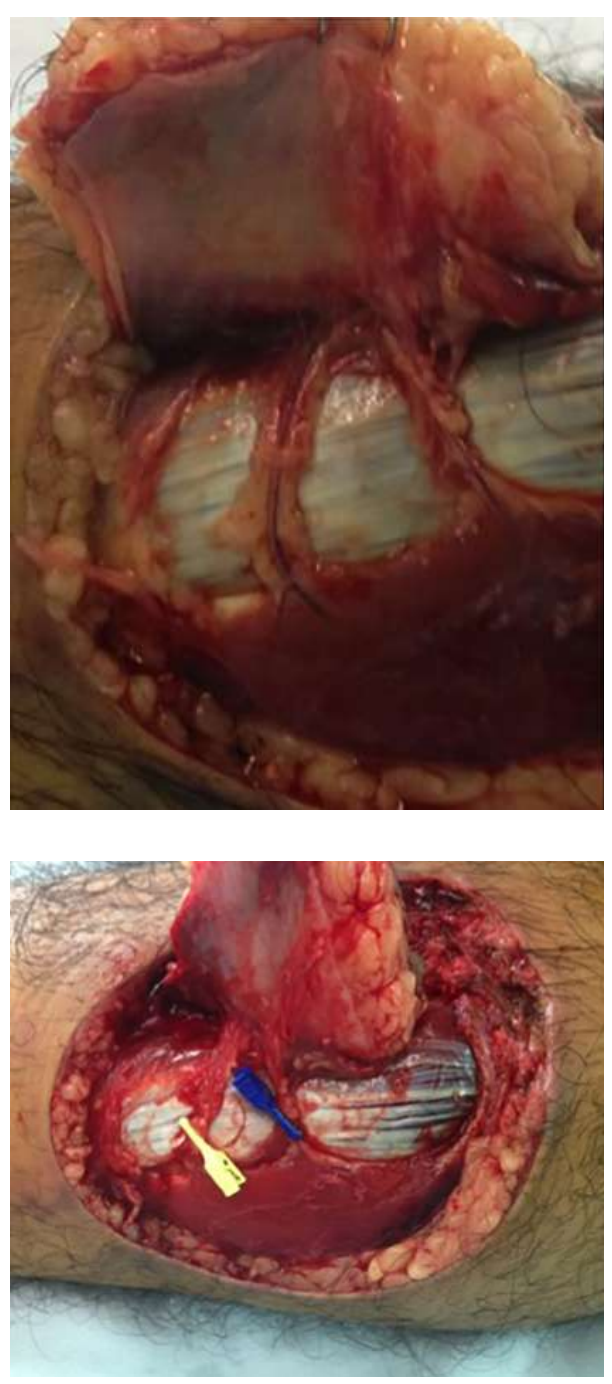

FIGURA 1. Disección y selección de la arteria perforante seleccionada 


\section{Análisis estadístico}

Se hizo un análisis descriptivo para obtener la frecuencia, la mediana y el rango intercuartílico de las variables estudiadas. Se estimaron los riesgos relativos entre la necrosis global (parcial más total), parcial o total, y la presencia de tabaquismo, hipertensión arterial sistémica, enfermedad arterial obstructiva crónica o diabetes mellitus, usando el test exacto de Fisher. Los datos se analizaron en SPSS ${ }^{\circledR}$, versión 22.

\section{Resultados}

Se realizaron 37 colgajos en 31 pacientes. La mediana de la edad fue de 40 años (rango intercuartílico: 26 a 64 años; rango: 18 a 82 años). El $77 \%$ de los pacientes eran hombres. La etiología más frecuente de los defectos de cobertura fue el trauma secundario a accidente en motocicleta (32\%). La descripción completa de la etiología se muestra en la tabla 1.

Se presentaron múltiples comorbilidades; las más comunes fueron hipertensión arterial sistémica (29\%), diabetes mellitus (19\%) y la enfermedad arterial oclusiva crónica (6\%). El $16 \%$ de los pacientes eran fumadores. El puntaje de la clasificación de la ASA fue de 2 , en $51 \%$ de los pacientes, de 1 , en el $28 \%$, y de 3 , en el $21 \%$.

La región más frecuentemente afectada fue la pierna (59\%), seguida de la región pélvica (22\%), la rodilla $(10 \%)$, el pie $(6 \%)$ y el muslo $(3 \%)$. Con respecto a la pierna, el lugar más afectado fue el tercio medio (54\%), seguido del tercio distal (28\%) y del tercio proximal (18\%). El tamaño promedio de los defectos de los tejidos blandos fue de $40,3 \mathrm{~cm}^{2}$.

El colgajo de arteria perforante más usado fue el de la arteria tibial posterior (38\%), seguido del de la arteria

TABLA 1.

Etiología del defecto de los tejidos blandos

\begin{tabular}{lc}
\hline Etiología & n (\%) \\
\hline Trauma & $12(32)$ \\
Úlceras por presión & $8(22)$ \\
Osteomielitis & $7(19)$ \\
Complicaciones de cirugías previas & $6(16)$ \\
Otros & $4(11)$ \\
\hline
\end{tabular}

peronea (16\%), el de la arteria glútea superior (14\%) y el de la arteria tibial anterior (8\%). La descripción completa de los colgajos utilizados se presenta en la tabla 2. El tamaño promedio de los colgajos fue de $52,5 \mathrm{~cm}^{2}$ y el arco de rotación estuvo entre los 90 y los $180^{\circ}$. Se logró el cierre primario del sitio donante en el $38 \%$ de los pacientes; los demás requirieron injerto de piel. El tiempo promedio de seguimiento fue de 137 días.

De los 37 colgajos realizados, dos (5\%) presentaron necrosis total y $9(24 \%)$ casos presentaron necrosis parcial; 5 (56\%) de estos últimos requirieron reintervención para lograr la cobertura con injerto de piel. Incluyendo las cirugías de revisión, se logró cobertura efectiva en el $100 \%$ de los pacientes.

Después del análisis estadístico, se encontró que los pacientes con enfermedad arterial oclusiva crónica (riesgo relativo, $\mathrm{RR}=5,67 ; \mathrm{p}=0,011$ ) y aquellos con ASA $3(p=0,019)$, presentaron mayor riesgo de desarrollar necrosis parcial; cuando se hizo el análisis sumando los casos de necrosis parcial y total (necrosis global), se encontró que tenían mayor riesgo los pacientes con hipertensión arterial sistémica $(R R=2,84 ; p=0,042)$ y con enfermedad arterial oclusiva crónica $(R R=4,25$; $\mathrm{p}=0,021)$; dichas diferencias fueron estadísticamente significativas (tablas 3 y 4 ).

\section{Caso 8}

\section{Casos representativos}

Se trata de un paciente de sexo masculino de 66 años de edad, que ingresó al hospital con diagnóstico de ruptura de aneurisma de la aorta abdominal. Presentaba ante-

TABLA 2.

La nomenclatura de los colgajos de arteria perforante incluye su eje vascular fuente ${ }^{5}$

\begin{tabular}{lc}
\hline Ejes vasculares & \% \\
\hline Arteria tibial posterior & 38 \\
Arteria peronea & 16 \\
Arteria glútea superior & 14 \\
Arteria tibial anterior & 8 \\
Arteria circunfleja medial femoral & 8 \\
Arteria glútea inferior & 6 \\
Arteria genicular superior lateral & 5 \\
Arteria primera metatarsiana dorsal & 5 \\
Estilo libre & 3 \\
\hline
\end{tabular}


TABLA 3.

Necrosis parcial

\begin{tabular}{lccccc}
\hline Variable & $\begin{array}{c}\text { No } \\
\text { expuesto } \\
\mathbf{n}(\%)\end{array}$ & $\begin{array}{c}\text { Necrosis } \\
\text { parcial } \\
(\mathbf{n}=\mathbf{9}) \\
\mathbf{n}(\mathbf{\%})\end{array}$ & $\begin{array}{c}\text { Riesgo } \\
\text { relativo }\end{array}$ & IC 95 \% & $\mathbf{p}$ \\
\hline Tabaquismo & $7(23)$ & $1(17)$ & 0,71 & $0,11-4,79$ & 0,59 \\
HTA & $4(15)$ & $5(46)$ & 2,96 & $0,97-8,96$ & 0,066 \\
EAOC & $6(18)$ & $3(100)$ & 5,67 & $2,74-11,71$ & 0,011 \\
DM & $6(21)$ & $3(38)$ & 1,81 & $0,58-5,69$ & 0,29 \\
ASA & & & & & 0,019 \\
2 & $1(13)$ & $2(11)$ & & & \\
3 & $1(13)$ & $5(63)$ & & & \\
\hline
\end{tabular}

HTA: hipertensión arterial sistémica; EAOC: enfermedad arterial oclusiva crónica; DM: diabetes mellitus; ASA: American Society of Anesthesiologists

TABLA 4.

Necrosis global (parcial más total)

\begin{tabular}{lccccc}
\hline Variable & $\begin{array}{c}\text { No } \\
\text { expuesto } \\
\text { n (\%) }\end{array}$ & $\begin{array}{c}\text { Necrosis global } \\
(\mathbf{n = 1 1 )} \\
\mathbf{n}(\%)\end{array}$ & $\begin{array}{c}\text { Riesgo } \\
\text { relativo }\end{array}$ & IC 95 \% & $\mathbf{p}$ \\
\hline Tabaquismo & $8(27)$ & $2(33)$ & 1,25 & $0,35-4,49$ & 0,54 \\
HTA & $5(19)$ & $6(55)$ & 2,84 & $1,09-7,37$ & 0,042 \\
EAOC & $8(23)$ & $3(100)$ & 4,25 & $2,32-7,79$ & 0,021 \\
DM & $8(28)$ & $3(38)$ & 1,36 & $0,47-3,97$ & 0,44 \\
ASA & & & & & \\
2 & $2(25)$ & $3(17)$ & & & \\
3 & $2(25)$ & $5(63)$ & & & \\
\hline
\end{tabular}

HTA: hipertensión arterial sistémica; EAOC: enfermedad arterial oclusiva crónica; DM: diabetes mellitus; ASA: American Society of Anesthesiologists

cedentes personales de hipertensión arterial sistémica, diabetes mellitus, obesidad y malnutrición.

Se trató quirúrgicamente y su posoperatorio se supervisó en la unidad de cuidados intensivos. Presentó una evolución tórpida, con estancia prolongada en la unidad de cuidados intensivos; desarrolló una úlcera sacra por presión que se sobreinfectó. Se le practicó desbridamiento quirúrgico y recibió antibióticos. El defecto sacro resultante fue de $18 \times 7 \mathrm{~cm}$, el cual se cubrió con dos colgajos bilaterales de la arteria glútea superior, y se permitió el cierre primario de ambos sitios donantes. Uno de los colgajos presentó congestión venosa de la punta, sin que se requirieran procedimientos adicionales. El periodo final de seguimiento fue de 145 días (figura 2).
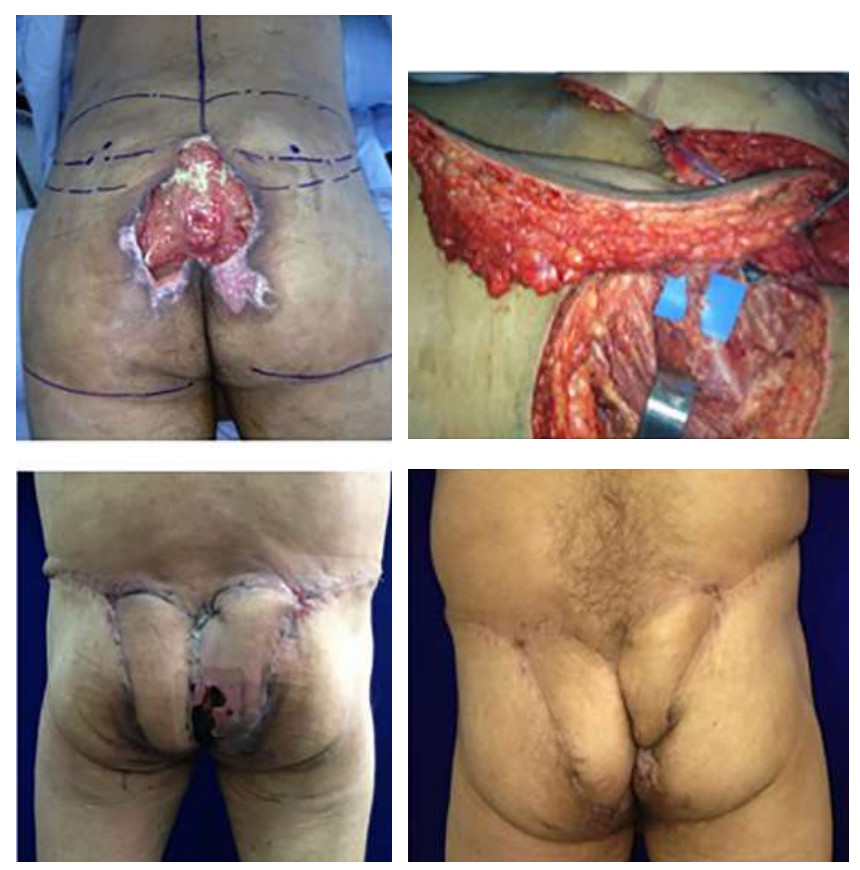

Figura 2. Caso 8. Arriba, a la izquierda: defecto preoperatorio; a la derecha: colgajo de arteria glútea superior; abajo, a la izquierda: necrosis menor; a la derecha: resultado después del proceso final de cicatrización

\section{Caso 21}

Se trata de un paciente de sexo masculino de 22 años de edad, que sufría osteomielitis crónica en la tibia derecha con múltiples reactivaciones previas, y había sido tratado previamente mediante cubrimiento con colgajo sural y colgajo peninsular medial fasciocutáneo al azar.

Presentó un nuevo defecto de tejidos blandos en la cara anterior del tercio medio de la misma pierna comprometida, que se trató con desbridamiento y antibioticoterapia. El tamaño del defecto era de $4 \times 3 \mathrm{~cm}$, por lo cual se cubrió con un colgajo de arteria circunfleja medial femoral. El sitio donante requirió injerto de piel de espesor parcial. Durante el seguimiento, el colgajo presentó congestión venosa del $30 \%$, la cual se resolvió sin procedimientos adicionales (figura 3 ).

\section{Caso 18}

Se trata de un paciente de sexo masculino de 72 años de edad, con antecedentes de osteomielitis crónica con múltiples reactivaciones. Se decidió practicar un desbridamiento adecuado y se inició el tratamiento con antibióticos. 
El defecto de cobertura final fue de $10 \times 8 \mathrm{~cm}^{2}$ sobre el tercio distal de la pierna y se reconstruyó con un colgajo de arteria tibial posterior. No se presentaron complicaciones vasculares. Después de 220 días de seguimiento, el resultado funcional y estético era muy satisfactorio (figura 4).

\section{Discusión}

Con respecto a la reconstrucción del miembro inferior y, en especial, de los defectos de los tejidos blandos loca-
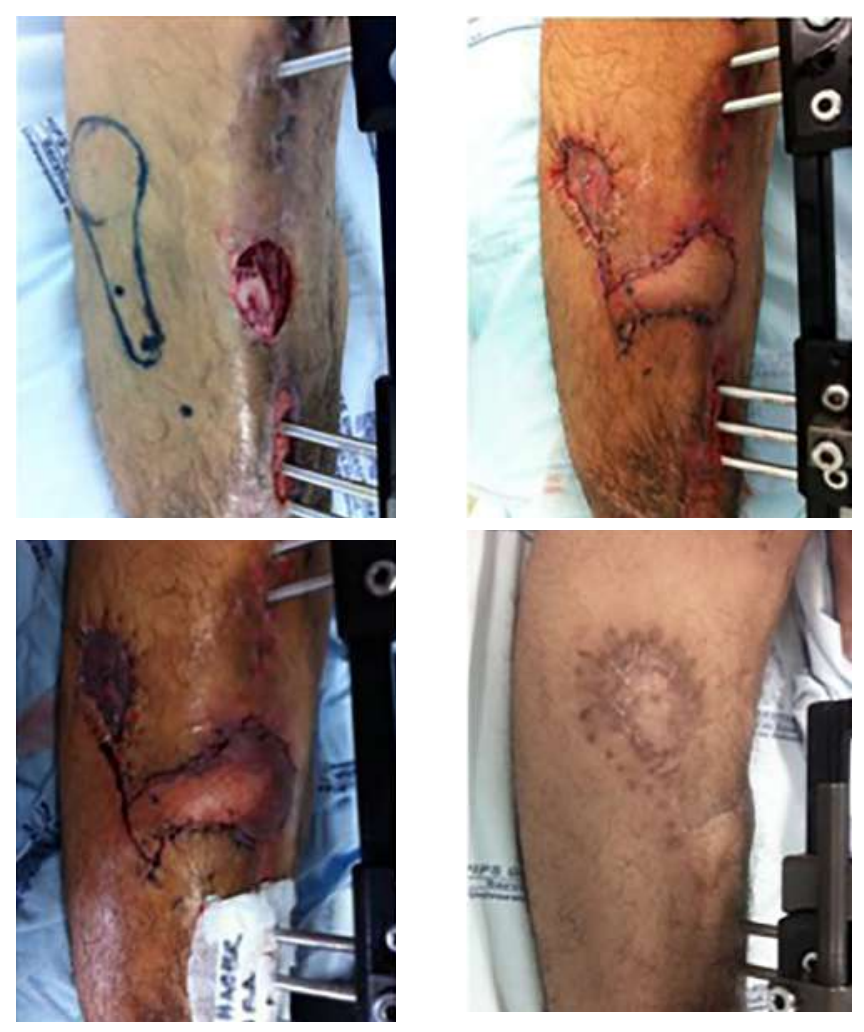

Figura 3. Caso 21. Arriba, a la izquierda: defecto preoperatorio; a la derecha: colgajo de la arteria tibial anterior; abajo, a la izquierda: congestión venosa distal; a la derecha: resultado final después de ocho meses de seguimiento lizados en el tercio inferior de la pierna, actualmente el cirujano plástico tiene múltiples opciones, pero no por esto menos desafíos. Durante la toma de decisiones, el primer aspecto que se debe considerar es la elección entre colgajos regionales (tradicionales o de arterias perforantes) o libres ${ }^{7}$. La tasa de complicaciones del miembro inferior para cada uno de ellos puede ser considerable y muy variable, dependiendo de la serie.

En múltiples publicaciones se ha revisado la anatomía vascular del miembro inferior, describiendo todos los territorios con las posibles arterias perforantes en las que se podrían basar los colgajos en hélice ${ }^{8-10}$. Además de la presencia de arterias perforantes en el sitio donante deseado, su calidad es la que realmente garantiza una adecuada perfusión del colgajo. La arteria perforante ideal debe tener, por lo menos, $1 \mathrm{~mm}$ de diámetro y de 20 a $30 \mathrm{~mm}$ de longitud ${ }^{11}$.

Durante la toma de decisiones en el proceso reconstructivo, la primera pregunta que debemos hacernos es: ¿por qué elegir un colgajo de arteria perforante? Los colgajos de arteria perforante tienen múltiples ventajas, a saber: 1) la versatilidad en el diseño y el amplio rango de rotación; 2) la menor morbilidad del sitio donante; 3) la calidad de los tejidos (textura, pigmentación y volumen) es muy similar a la del sitio receptor; 4) no se sacrifican ejes vasculares mayores, y 5) cuando se alcanza la curva de aprendizaje adecuada, la disección es fácil, rápida y de menor costo, comparada con la de los colgajos libres ${ }^{12-14}$.

El otro aspecto que se debe considerar son los límites en las indicaciones de los colgajos en hélice de las arterias perforantes. Según nuestra experiencia, cuando la extensión del defecto que se debe cubrir es mayor de $15 \mathrm{~cm}$ de longitud, existe un riesgo importante de necrosis distal; esto es un problema ya que, usualmente,
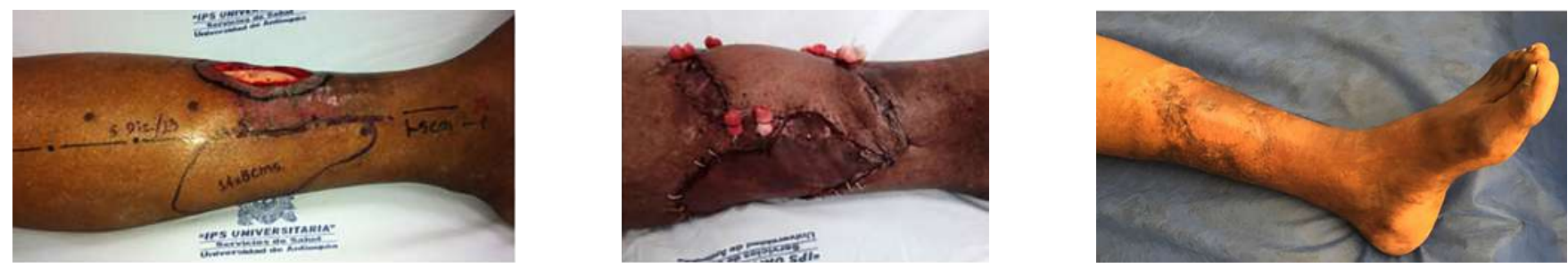

FIgURA 4. Caso 18: a la izquierda: úlcera crónica; en el centro: colgajo de arteria tibial posterior; a la derecha: después de 220 días de seguimiento, con excelente resultado funcional y estético. 
se localiza en una zona crítica del defecto por cubrir. Asimismo, en los defectos distales de cubrimiento del pie, como aquellos localizados en el talón, se presentan ciertas dificultades, ya que por su lejanía se necesita mayor arco de rotación y mayor tamaño de las islas de piel para alcanzar el sitio del defecto; por lo tanto, la posibilidad de que se presente necrosis parcial en estos casos se incrementa y la morbilidad del sitio donante, también.

Las tasas de falla terapéutica con los colgajos libres en la reconstrucción del miembro inferior varían según la serie entre 4 y $19 \%$, y la tasa de complicaciones global está entre 16 y $38 \%{ }^{15-17}$. Cuando se revisan las series de colgajos regionales tradicionales, como el colgajo sural, se encuentran tasas de complicaciones globales de hasta $59 \%$ y, para las complicaciones vasculares, la tasa de necrosis total es de hasta $19 \% \mathrm{y}$, la de la parcial, hasta de $17 \%{ }^{18}$. En presencia de comorbilidades, como diabetes mellitus, enfermedad arterial oclusiva crónica e insuficiencia venosa, la tasa de necrosis puede ser de $33 \%{ }^{19}$. Con respecto a las tasas reportadas de falla terapéutica con los colgajos de arterias perforantes, la necrosis parcial varía entre 3,8 y $37,5 \%$, y la necrosis total, entre 3,8 y $17 \%{ }^{19-21}$.

En la presente serie, la tasa de necrosis parcial fue de $25 \%$; el $56 \%$ de estos casos requirió una nueva cirugía para lograr el cubrimiento completo, y la tasa de necrosis total fue de $5 \%$. Incluyendo las cirugías de revisión, se logró una cobertura efectiva del 100 $\%$ de los pacientes. Se encontró una asociación estadísticamente significativa de la hipertensión arterial sistémica y la enfermedad arterial obstructiva crónica con la necrosis; además, los pacientes con ASA 3 presentaron mayor tasa de complicaciones. Como era de esperarse, entre más comorbilidades tenga el paciente, es más probable que presente complicaciones y la edad, en forma aislada, podría no ser un factor limitante. Dependiendo de la serie que se revise, se puede encontrar gran variabilidad en la tasa reportada de complicaciones, lo cual podría explicarse por las diferentes variables que podrían influir en el resultado, tales como el tipo de paciente y sus diferentes comorbilidades.

Consideramos que este estudio muestra por primera vez la experiencia local, y tiene como ventaja el haber incluido pacientes muy heterogéneos, de diferentes edades y con distintas comorbilidades, lo cual demuestra que los colgajos en hélice de las arterias perforantes son una alternativa segura y muy versátil, teniendo en cuenta que, a mayor número de comorbilidades, es mayor la probabilidad de complicaciones. La desventaja de este estudio es su diseño retrospectivo y el número de pacientes, lo cual puede generar sesgos en la selección al recolectar toda la información deseada.

\section{Conclusión}

Los colgajos en hélice de las arterias perforantes son una opción confiable de cubrimiento para los defectos de tejidos blandos del miembro inferior, con tasas de complicaciones similares a las de otros métodos tradicionales. Las comorbilidades, como la hipertensión arterial sistémica y la enfermedad arterial oclusiva crónica, y un puntaje de 3 en la clasificación ASA, se asociaron con un mayor riesgo de necrosis en este estudio y deben ser tenidas en cuenta en la elección del procedimiento que se vaya a realizar.

\title{
Helicoidal flap of perforating arteries in reconstruction of resection defects in the lower extremity: experience at the University IPS, Medellín, Colombia
}

\author{
Abstract \\ Introduction: Pedicled propeller perforator flaps are a versatile local reconstructive option for defects of the lower \\ leg, ankle and foot. \\ Material and methods: A retrospective review of 37 cases undergoing this procedure in the period 2012 to 2015 was \\ performed. The analysis includes demographic and perioperative factors, complications and outcomes. Thirty-seven \\ flaps were performed on a single perforator from any of the major vascular axes of the lower extremity.
}


Results: The mean age was 44.3 years (range 18 -82 years). Etiology of soft tissue defects was trauma in 32\% of the cases, but osteomyelitis, pressure sores and vascular ulcers were also present. Pedicle rotation arch ranged between 90 to 180 degrees. There was 5\% percent complete necrosis and $24 \%$ partial flap failure rate, both associated with arteriopathy and ASA 3 score.

Conclusion: Propeller perforator flaps provide reliable coverage for lower limb defects, with a comparable rate of complications to traditional regional flaps.

Key words: Perforator flap; lower extremity; wounds and injuries; comorbidity; postoperative complications; necrosis.

\section{Referencias}

1. Demirtas Y, Kelahmetoglu O, Cifci M, Tayfur V, Demir A, Guneren E. Comparison of free anterolateral thigh flaps and free muscle-musculocutaneous flaps in soft tissue reconstruction of lower extremity. Microsurgery. 2010;30:24-31.

2. Meyer A, Goller K, Horch RE, Beier JP, Taeger CD, Arkudas A, et al. Results of combined vascular reconstruction and free flap transfer for limb salvage in patients with critical limb ischemia. J Vasc Surg. 2015;61:1239-48.

3. Fischer JP, Wink JD, Nelson JA, Cleveland E, Grover R, Wu LC, et al. A retrospective review of outcomes and flap selection in free tissue transfers for complex lower extremity reconstruction. J Reconstr Microsurg. 2013;29:407-16.

4. Masquelet AC, Romana MC, Wolf G. Skin island flaps supplied by the vascular axis of the sensitive superficial nerves: Anatomic study and clinical experience in the leg. Plast Reconstr Surg. 1992;89:1115-21.

5. Hyakusoku H, Yamamoto T, Fumiiri M. The propeller flap method. Br J Plast Surg. 1991;44:53-4.

6. Masià J, Clavero JA, Carrera A. Planificación preoperatoria de los colgajos de perforantes. Cir Plást Iberolatinoam. 2006;32:237-42.

7. Hupkens P, Schijns W, van Abeelen M, Kooloos JGM, Slater NJ, Ulrich DJO. Lateral lower leg perforator flaps: An anatomical study to localize and classify lateral lower leg perforators. Microsurgery. 2015;35:140-7.

8. Geddes C, Tang M, Yang D, Morris S. Anatomy of the integument of the lower extremity. In: Blondeel P, Morris S, Hallock G, Neligan P, editors. Perforator flaps anatomy, technique and clinical applications. St Louis, MO: Quality Medical Publishing; 2006. p. 541-77.

9. Morris SF, Tang M, Almutari K, Geddes C, Yang D. The anatomic basis of perforator flaps. Clin Plast Surg. 2010;37:553-70.

10. Morris SF, Tang M, Geddes CR. Bases anatómicas vasculares de los colgajos perforantes cutáneos. Cir Plást Iberolatinoam. 2006;32:225-36.

11. Lu TC, Lin CH, Lin CH, Lin YT, Chen RF, Wei FC. Versatility of the pedicled peroneal artery perforator flaps for soft-tissue coverage of the lower leg and foot defects. J Plast Reconstr Aesthet Surg. 2011;64:386-93.
12. Jack MC, Newman MI, Barnavon Y. Islanded posterior tibial artery perforator flap for lower limb reconstruction: Review of lower leg anatomy. Plast Reconstr Surg. 2011;127:1014-5.

13. Jiga LP, Barac S, Taranu G, Blidisel A, Dornean V, Nistor A, et $a l$. The versatility of propeller flaps for lower limb reconstruction in patients with peripheral arterial obstructive disease: Initial experience. Ann Plast Surg. 2010;64:193-7.

14. Georgescu AV, Matei IR, Capota IM. The use of propeller perforator flaps for diabetic limb salvage: A retrospective review of 25 cases. Diabet Foot Ankle. 2012:3.

15. Wong AK, Joanna Nguyen T, Peric M, Shahabi A, Vidar EN, Hwang BH, et al. Analysis of risk factors associated with microvascular free flap failure using a multi-institutional database. Microsurgery. 2015;35:6-12.

16. Bui DT, Cordeiro PG, Hu Q-Y, Disa JJ, Pusic A, Mehrara BJ. Free flap reexploration: Indications, treatment, and outcomes in 1193 free flaps. Plast Reconstr Surg. 2007;119:2092-100.

17. Gir P, Cheng A, Oni G, Mojallal A, Saint-Cyr M. Pedicled-perforator (propeller) flaps in lower extremity defects: A systematic review. J Reconstr Microsurg. 2012;28:595-601.

18. Follmar KE, Baccarani A, Baumeister SP, Levin LS, Erdmann D. The distally based sural flap. Plast Reconstr Surg. 2007;119:138e-48e.

19. Sameem M, Au M, Wood T, Farrokhyar F, Mahoney J. A systematic review of complication and recurrence rates of musculocutaneous, fasciocutaneous, and perforator-based flaps for treatment of pressure sores. Plast Reconstr Surg. 2012;130:67e-77e.

20. Nelson J, Fischer J, Brazio P, Kovach S, Rosson G, Rad A. A review of propeller flaps for distal lower extremity soft tissue reconstruction: Is flap loss too high? Microsurgery. 2013;33:578-86.

21. Lykoudis EG, Koutsouris M, Lykissas MG. Vascular anatomy of the integument of the lateral lower leg: An anatomical study focused on cutaneous perforators and their clinical importance. Plast Reconstr Surg. 2011;28:188-98.

Correspondencia: Carolina Posso, MD

Correo electrónico: carolina.posso@gmail.com Medellín, Colombia 